\title{
Inibidores da SGLT2 e suas influências no sistema cardiovascular: uma revisão sistemática
}

\author{
SGLT2 inhibitors and their influence on the cardiovascular system: a systematic review \\ Inhibidores de SGLT2 y sus influencias en el sistema cardiovascular: una revisión \\ sistemática
}

Guilherme Antoneli Silva1*, Rodolpho Gareti Carreira1, Wendell Felipe Garcia1, Aloísio Eugênio Cavasini Filho'.

\section{RESUMO}

Objetivo: Descrever, através de uma revisão sistemática, os principais estudos, novidades e influências dos inibidores da Sodium Glucose Cotransporter 2 (SGLT2) no sistema cardiovascular de pacientes diabéticos tipo 2. Métodos: Foram utilizados para pesquisa a base de dados BIREME, considerando textos com os descritores "Inibidores do Transportador 2 de Sódio-Glicose" e "Doenças Cardiovasculares", publicados entre o período dos anos de 2009-2019, redigidos em inglês, português e espanhol, sendo, posteriormente, avaliados, interpretados e tabulados. Resultados: Inicialmente, 277 artigos foram encontrados na pesquisa, sendo que 192 publicações foram descartadas da avalição por não evidenciarem os critérios de inclusão necessários, tendo sido selecionadas 85 publicações. Destes 85 artigos que contemplaram os critérios de seleção, 38 eram de acesso restrito aos assinantes de seus jornais e os demais 47 foram escolhidos e analisados pelos autores. Considerações finais: Conclui-se que a classe das medicações inibidoras da SGLT2 mostrou-se como opção terapêutica de grande interesse para diabéticos tipo 2, visto sua capacidade de promover inúmeras alterações sistêmicas benéficas nestes pacientes e influenciar diretamente na redução do risco cardiovascular, com redução nas taxas de mortalidade e hospitalização por insuficiência cardíaca.

Palavras-chave: Hipoglicemiantes, Sistema cardiovascular, Tratamento, Diabetes.

\begin{abstract}
Objective: To describe, through a systematic literature review, the main studies, news and influences of Sodium Glucose Cotransporter 2 (SGLT2) inhibitors on the cardiovascular system of type 2 diabetic patients. Methods: The BIREME database was used for research, considering texts with the descriptors "Sodium Glucose Transporter 2 Inhibitors" and "Cardiovascular Diseases", published between the years of 2009-2019, written in English, Portuguese and Spanish, subsequently evaluated, interpreted and tabulated. Results: Initially, 277 articles were found in the research. After review 192 publications were discarded from the evaluation for not having evidenced the necessary inclusion criteria, and 85 publications were selected. Of these 85 articles that presented the selection criteria, 38 had restricted use and access to their journals subscribers. The remaining 47 texts were analyzed and chosen by the authors for review. Final considerations: It is concluded that the medications of SGLT2 inhibitors are shown to be a therapeutic option of great interest for type 2 diabetics, given their capacity to promote numerous beneficial systemic changes in those patients and directly influence the reduction of cardiovascular risk, reducing the mortality and heart failure hospitalizations rates.
\end{abstract}

Key words: Hypoglycemic, Cardiovascular system, Treatment, Diabetes.

\footnotetext{
${ }^{1}$ Faculdade de Medicina da Universidade de Franca (UNIFRAN), Franca - SP.

*E-mail: guilhermeantoneli@msn.com
} 


\section{RESUMEN}

Objetivo: Describir a través de una revisión bibliográfica sistemática, los principales estudios, novedades e influencias de los inhibidores de Sodium Glucose Cotransporter 2 (SGLT2) en el sistema cardiovascular de pacientes con diabetes tipo 2. Métodos: Se utilizó la base de datos BIREME para la investigación, considerando textos con los descriptores "Inhibidores del transportador de sodio-glucosa 2" y "Enfermedades cardiovasculares", publicados entre los años de 2009-2019 y escritos en inglés, portugués y español, habiendo sido posteriormente evaluados, interpretados y tabulados. Resultados: Inicialmente, se encontraron 277 artículos en la investigación. Después de la revisión, se descartaron 192 publicaciones de la evaluación por no haber evidenciado los criterios de inclusión necesarios, y se seleccionaron 85 publicaciones. De estos 85 artículos que presentaron los criterios de selección, 38 tenían uso y acceso restringido a los suscriptores de sus periódicos. Los 47 textos restantes fueron analizados y elegidos para revisión por los autores. Consideraciones finales: Se concluye que la clase de medicamentos inhibidores de SGLT2 son una opción terapéutica de gran interés para los diabéticos tipo 2, dada su capacidad en promover numerosas alteraciones sistémicas benéficas en estos pacientes e influir directamente en la reducción en las tasas de mortalidad y hospitalización por insuficiencia cardíaca.

Palabras clave: Hipoglucemiantes, Sistema cardiovascular, Tratamiento, Diabetes.

\section{INTRODUÇÃO}

A Diabetes Mellitus tipo 2 (DM2) representa atualmente um grande problema de saúde mundial. Ao longo dos anos a incidência desta comorbidade vêm aumentando exponencialmente (OLIVEIRA JEP, et al., 2017). Inúmeros fatores sustentam a rápida progressão da incidência e prevalência da DM2 na população: urbanização, mudança de hábitos alimentares, sedentarismo, obesidade, tabagismo, histórico familiar, envelhecimento populacional e outros fatores atrelados ao crescimento populacional e ao estilo de vida moderno (LEY SH, et al., 2015).

Segundo dados da International Diabetes Federation (IFD) (2019) cerca de 463 milhões de pessoas entre 20 e 79 anos tinham diabetes, enquanto previsões estimam que em 2030 quase 578.4 milhões serão acometidos e em 2045, 700.2 milhões de pessoas terão diabetes no mundo.

Estima-se que o Brasil possua aproximadamente 16.8 milhões de adultos diabéticos, sendo o país com a maior prevalência de diabéticos na américa central e do sul. Nestas regiões geográficas, o Brasil arca com os maiores custos com a doença, tendo gastado em 2019 cerca de 3,117 bilhões de dólares ou 24,2\% do orçamento anual da saúde. Apesar dos grandes gastos, registrou-se em 2019 no país 135.200 mortes relacionadas à comorbidade (IDF, 2019).

Os elevados custos e grande capacidade de morbimortalidade induzida pela DM2 se dá pela capacidade de acometimento de múltiplos órgãos e sistemas. Destes, um dos mais acometidos é o cardiovascular, sofrendo danos micro e macrovasculares contínuos devido à hiperglicemia, o que torna a glicemia elevada um fator de risco importante para o desenvolvimento e agravo de doenças cardiovasculares (SOCIEDADE BRASILEIRA DE DIABETES, 2014). Além de fator de risco para o desenvolvimento de doenças cardiovasculares, a diabetes confere maior risco de hospitalização por insuficiência cardíaca (IC), acontecimento de eventos isquêmicos e morte cardiovascular.

Dados publicados em 2015 na revista Metabolic Syndrome and Related Disorders, apontam que nos Estados Unidos da América (EUA), 30\% dos diabéticos tipo 2 irão desenvolver IC ao longo da vida e das hospitalizações por IC, 40\% são portadores de DM2, ratificando a relação entre diabetes e o aumento do risco cardiovascular (RIGGS K, et al., 2015).

Com a elucidação do aumento do risco cardiovascular devido à diabetes e estudos constatando que hipoglicemiantes altamente empregues no tratamento da DM2 como a glitazonas, sulfoniluréias e insulinas aumentavam o risco cardiovascular (PANCHOLIA AK, 2018), a Food and Drugs Administration (FDA) tornou obrigatório no ano de 2008 o desenvolvimento de estudos para constatação do não agravamento do risco cardiovascular induzido por hipoglicemiantes (CEFALU WT, et al., 2018). 
Anteriormente à obrigatoriedade da FDA, as classes de drogas disponíveis para o tratamento da DM2 eram as biguanidas, sulfoniluréias, metiglinidas, inibidores da alfaglicosidase, glitazonas e insulinas (SOCIEDADE BRASILEIRA DE DIABETES, 2014).

Por anos tais medicações tiveram grande emprego médico, no entanto, após 2008 e com a introdução do conceito do aumento do risco cardiovascular iatrogênico, o uso de algumas medicações passou a sofrer duras críticas, possuírem indicações e contraindicações específicas e concorrência de medicações mais modernas (YEHYA A e SADHU AR, 2018; SCHNELL O, et al., 2017).

Os Inibidores da Dipeptidil Peptidase-4 (DPP4), são capazes de mimetizar as incretinas pancreáticas, e foram um dos primeiros medicamentosas a seguir tal regulamentação da FDA (YEHYA A e SADHU AR, 2018; FISMAN EZ e TENENBAUM A, 2015).

O primeiro representante dessa classe foi a Saxagliptina, que através do estudo Saxagliptin Assessment of Vascular Outcomes Recorded in Patients with Diabetes Mellitus-Thrombolysis in Myocardial Infarction 53 (SAVOR-TIMI 53) constatou risco aumentado de hospitalização por IC atrelado ao uso da droga.

Outros estudos foram conduzidos com inibidores da DPP4, como o estudo da Alogliptina, Examination of Cardiovascular Outcomes with Alogliptin Versus Standard of Care (EXAMINE), estudo da Sitagliptina, Trial Evaluating Cardiovascular Outcomes with Sitagliptin (TECOS) e o com a Linagliptina, Cardiovascular and Renal Microvascular Outcome Study With Linagliptin in Patients With Type 2 Diabetes Mellitus (CARMELINA). Todos esses estudos demonstraram neutralidade das drogas no sistema cardiovascular, não demonstrando piora ou melhora dos parâmetros com seu emprego (FISMAN EZ e TENENBAUM A, 2015).

Outra classe hipoglicemiante a seguir as normas da FDA foram os análogos da Glucagon-Like Peptide-1 (GLP1). Assim como os inibidores da DPP4, são medicações que mimetizam as incretinas pancreáticas, mas com tempo de ação mais prolongado e melhor efeito no controle glicêmico.

Uma das principais drogas representantes desta classe é a Liraglutida, que diferentemente dos estudos conduzidos com os inibidores da DPP4, constatou através do estudo Liraglutide Effect and Action in Diabetes: Evaluation of Cardiovascular Outcome Results (LEADER) redução de eventos cardiovasculares como infarto agudo do miocárdio (IAM), acidente vascular encefálico (AVE) e mortalidade de todas as causas, com a administração em pacientes com DM2 e risco cardiovascular preexistente (MANN JFE, et al., 2018).

Os dados publicados explicitando a ausência de efeitos positivos sobre o risco cardiovascular de inúmeras medicações hipoglicemiantes empregues no meio médico para o tratamento da DM2 (PANCHOLIA AK, 2018; FISMAN EZ e TENENBAUM A., 2015) tornou a Liraglutida em 25 de Janeiro de 2010, após o estudo LEADER (MANN JFE, et al., 2018), a primeira droga hipoglicemiante autorizada à comercialização pela FDA, comprovadamente efetiva na redução do risco cardiovascular (FOOD AND DRUG ADMINISTRATION, 2010).

Posteriormente à repercussão gerada com o potencial terapêutico dos análogos da GLP1, os inibidores da SGLT2 obtiveram grande destaque mundial com resultados surpreendentes no tratamento de pacientes com DM2 (PANCHOLIA AK, 2018; ZINMAN B, et al., 2015).

Esses resultados, encabeçados pelo estudo da Empaglifozina, nomeado The Empagliflozin Cardiovascular Outcome Event Trial in Type 2 Diabetes Mellitus Patients-Removing Excess Glucose (EMPAREG), demonstrou a capacidade dos inibidores da SGLT2 em diminuir o risco cardiovascular de pacientes com DM2, reduzindo a probabilidade de IAM (GILBERT RE e CONNELY KA, 2019), mortalidade geral e cardiovascular e hospitalização por IC.

Além dos parâmetros cardiovasculares, os inibidores da SGLT2 também se mostraram eficazes na redução da pressão arterial, do peso e outros parâmetros melhores discutidos posteriormente (HEERSPINK HJL, et al., 2016; ZINMAN B, et al., 2015; FOOTE C, et al., 2012).

Diante dessa problemática e com o destaque atual dos inibidores da SGLT2 devido às suas repercussões cardiovasculares e em outros inúmeros parâmetros, este trabalho têm como finalidade, através de uma revisão bibliográfica sistemática, analisar as principais e mais atualizadas fontes e estudos de como os inibidores da SGLT2 influenciam a redução do risco cardiovascular na DM2. 


\section{MÉTODOS}

Este estudo teve como objetivo a revisão sistemática das repercussões do uso de inibidores da SGLT2 no sistema cardiovascular. O processo de elaboração do projeto foi dividido em quatro etapas distintas. A primeira etapa se deu com a revisão literária, no qual foi utilizado a base de dados do Centro Latino-Americano de Informação em Ciências da Saúde, Biblioteca Regional de Medicina (BIREME). Para a seleção da bibliografia foram consideradas como palavras-chaves "Inibidores do Transportador 2 de Sódio-Glicose" e "Doenças Cardiovasculares".

Somente textos com tais descritores foram selecionados para avaliação individual pelos autores. Além do mais, seguiu-se como fatores de inclusão: (1) Apenas textos completos; (2) Textos em inglês, português e espanhol; (3) Publicações entre 2009 - 2019; (4) Menção às repercussões no sistema cardiovascular pela utilização dos inibidores da SGLT2 no título ou resumo. Na segunda etapa, os textos selecionados foram classificados em "relevantes", "parcialmente relevantes" e "não relevantes". Em casos de dúvida dos autores quanto à classificação, recorria-se à opinião do orientador. A terceira etapa visou o levantamento e tabulação dos dados e informações mais pertinentes. Os artigos selecionados conforme as duas primeiras etapas, foram avaliados e redigidos com auxílio do software Microsoft Office ${ }^{\circledR} 2016$ e o plugin da Mendeley (C) para organização das fontes bibliográficas. A quarta e última etapa destinou-se aos resultados e à discussão.

\section{RESULTADOS}

Após pesquisa das referências na base de dados BIREME, foram encontrados 277 artigos utilizando as palavras-chaves descritas, sendo que 192 deles não apresentavam os critérios de inclusão e 38 que contemplavam os critérios necessários eram de acesso restrito aos assinantes de suas respectivas revistas de publicação. Ao final desta primeira etapa de seleção bibliográfica, 47 artigos foram selecionados (Figura 1).

Figura 1 - Processo de seleção das referências.

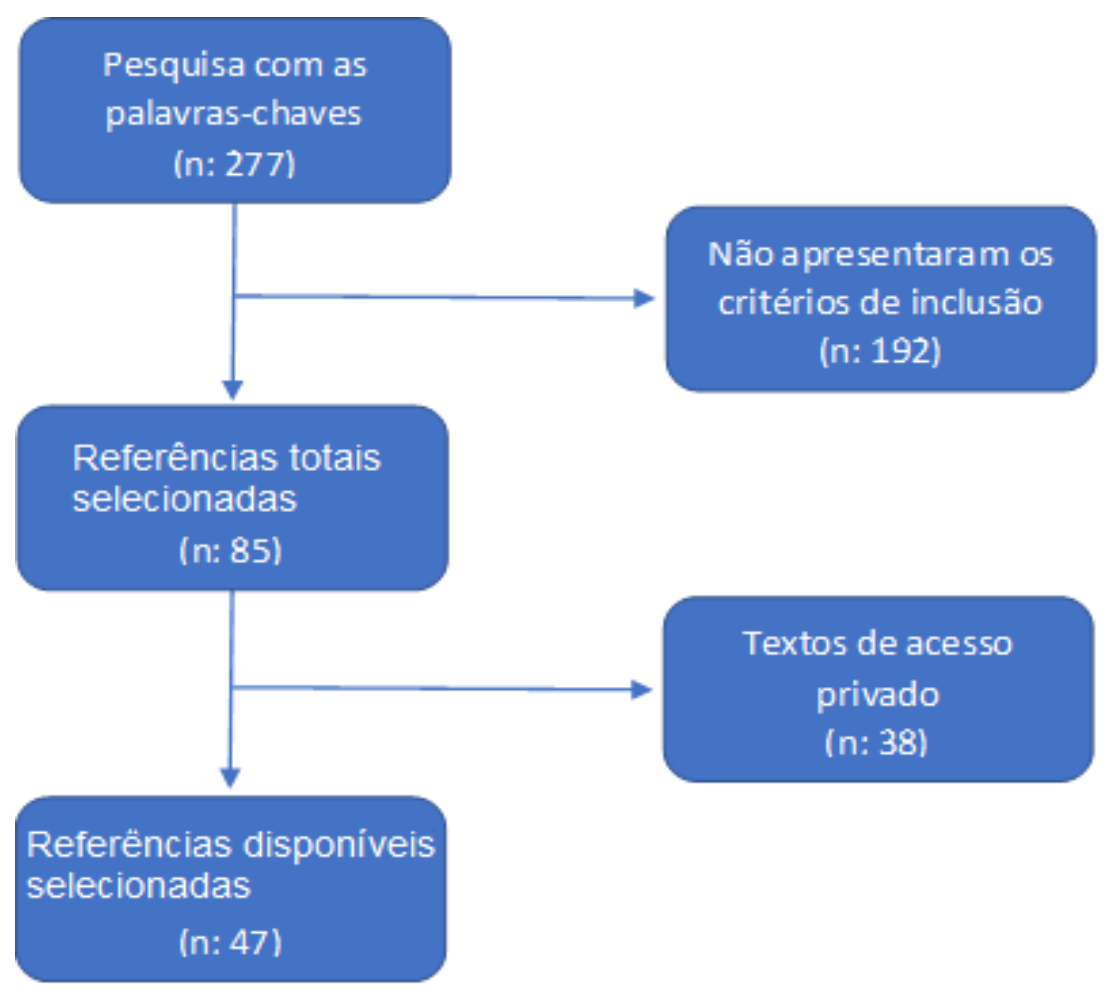

Fonte: Silva GA, et al., 2020. 
Das 47 referências inicialmente selecionadas, a maioria dos artigos foram publicados nos Estados Unidos da América (EUA), com um total de 17 publicações ou 36,2\% do total; em segundo lugar com 5 publicações está o Canadá, equivalente à 10,6\%; em terceiro lugar com 4 publicações cada ou $8,53 \%$ dos textos observamos Japão e Itália; o Reino Unido, com 3 publicações ou 6,39\% ficou em quarto lugar. Alemanha, Holanda, Suécia, Austrália e China apresentaram 2 artigos cada ou 4,24\%; e por último, Dinamarca, França, Vaticano e Índia com apenas 1 publicação cada ou 2,13\% de todas as publicações (Gráfico 1).

Gráfico 1 - Quantidade de artigos publicados por cada país.

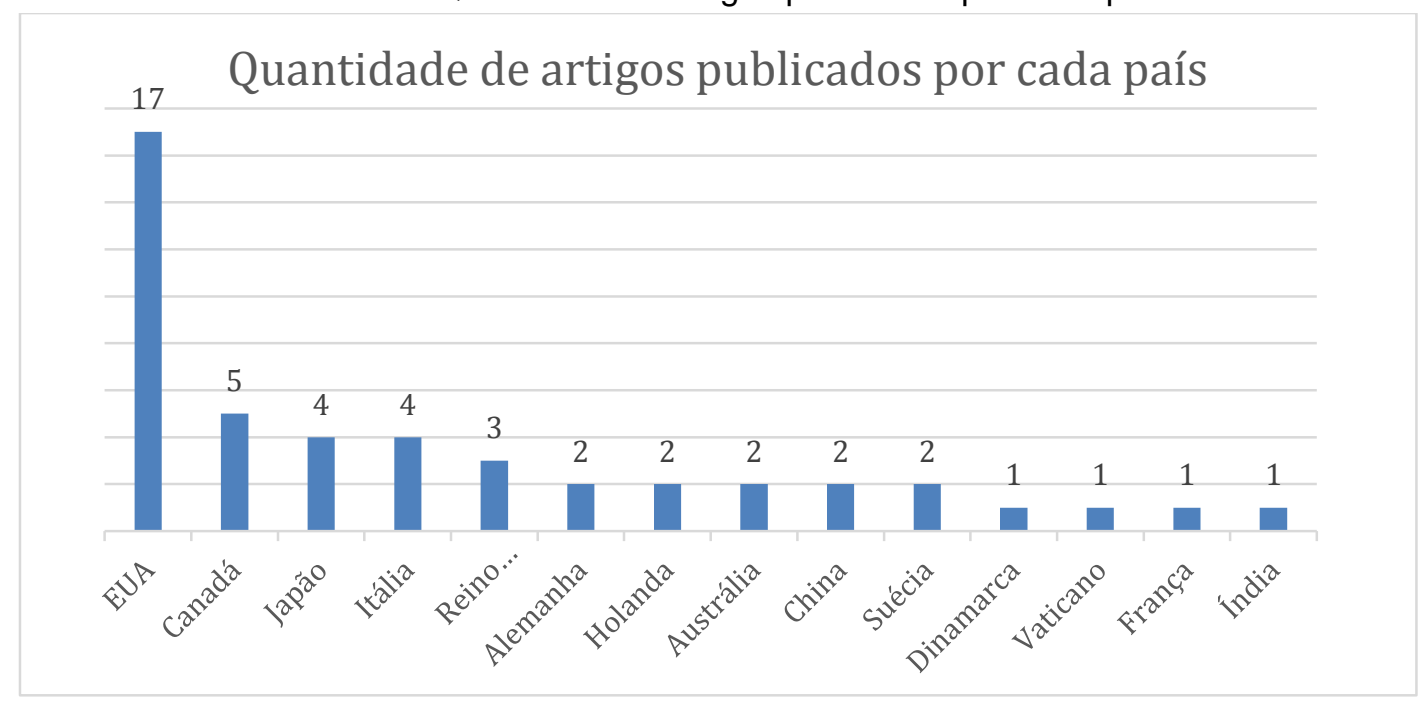

Fonte: Silva GA, et al., 2020.

Quando considerado o ano de publicação dos textos selecionados, a maioria dos artigos foi publicada no ano de 2017, sendo 18 ao todo. Em seguida, 2019 com 9 artigos, $2018 \mathrm{com} \mathrm{8,} 2016 \mathrm{com} \mathrm{4,} 2015 \mathrm{com} 4$ e 2011, 2012, 2013 e 2014 com 1 publicação cada. Os anos de 2009 e 2010 não apresentaram nenhuma publicação selecionada (Gráfico 2).

Gráfico 2 - Quantidade de artigos por ano de publicação.

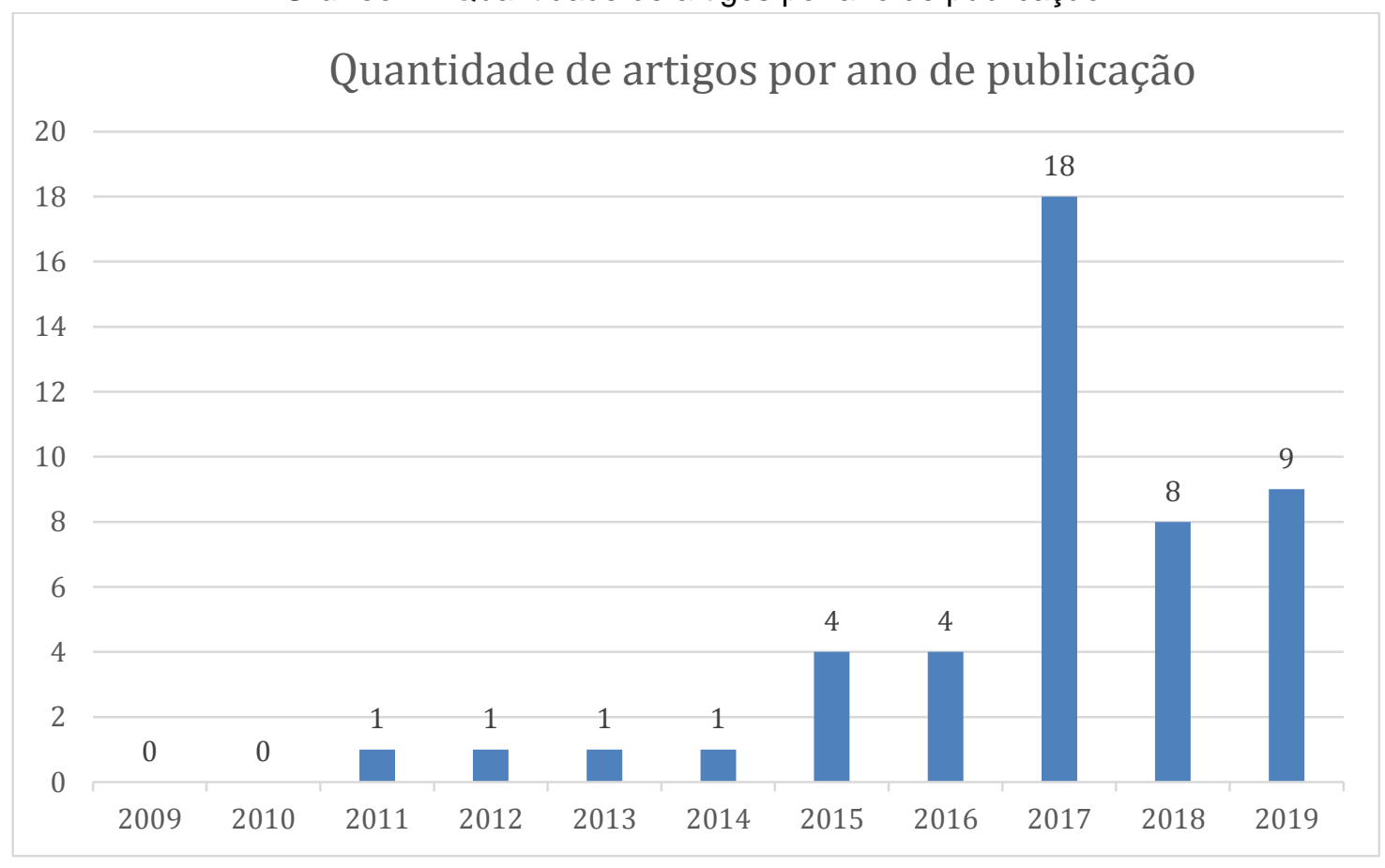

Fonte: Silva GA, et al., 2020. 
Quanto ao idioma das publicações selecionados, apesar da inclusão na pesquisa do português e espanhol, todas as 47 referências, $100 \%$ da seleção, se apresentaram redigidas em inglês (Gráfico 3).

Gráfico 3 - Idioma dos artigos selecionados

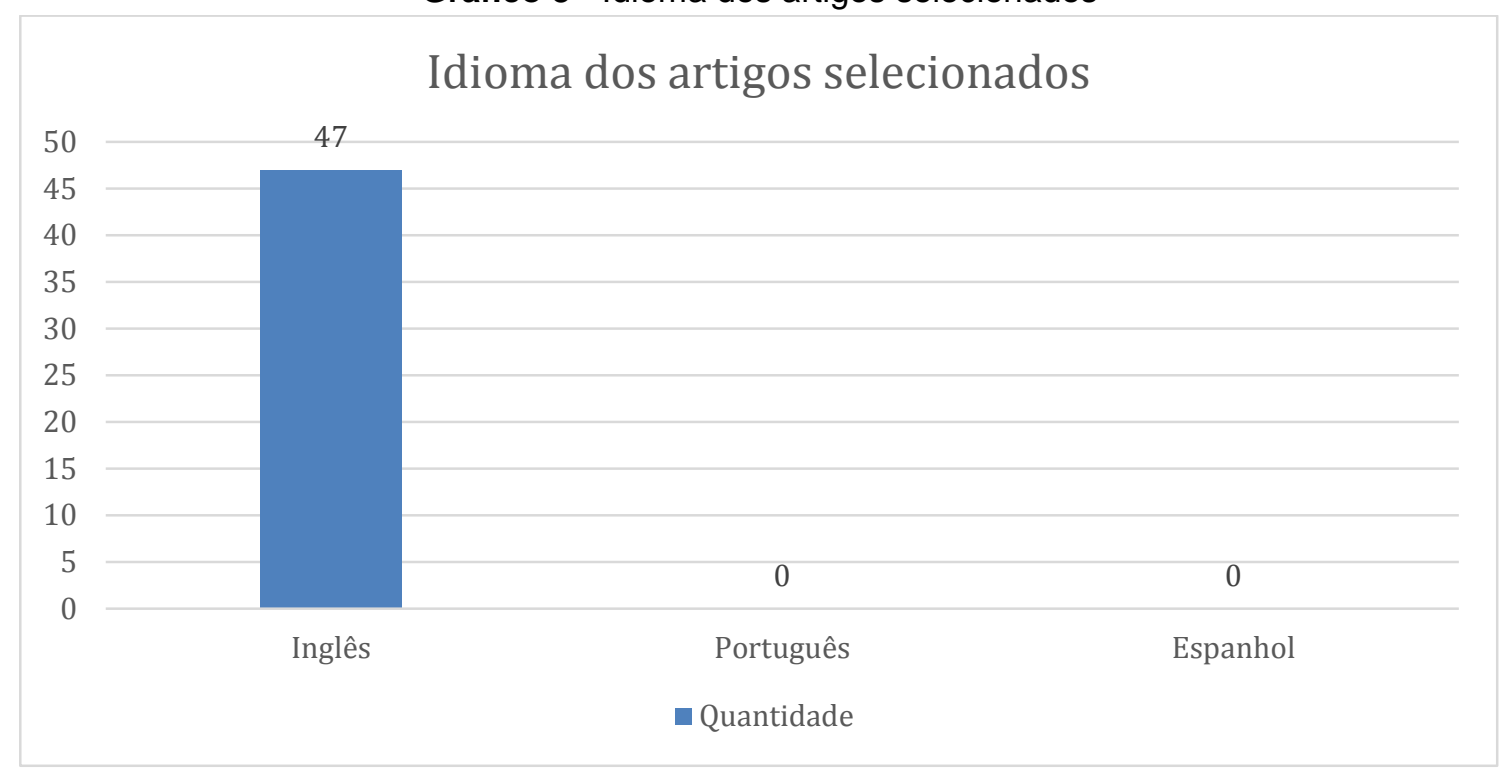

Fonte: Silva GA, et al., 2020.

Após, as referências foram lidas individualmente e divididas em três classificações distintas. Cinco textos foram classificados como "Parcialmente relevantes" tendo seu uso resguardado caso informações adicionais fossem necessárias para a elaboração da discussão, sete artigos determinados como "Não relevantes" e excluídos devido à resultados e informações finais duvidosas e por fim, 35 referências classificadas como "Relevantes" e selecionadas para a pesquisa (Figura 2).

Figura 2 - Seleção final das referências.

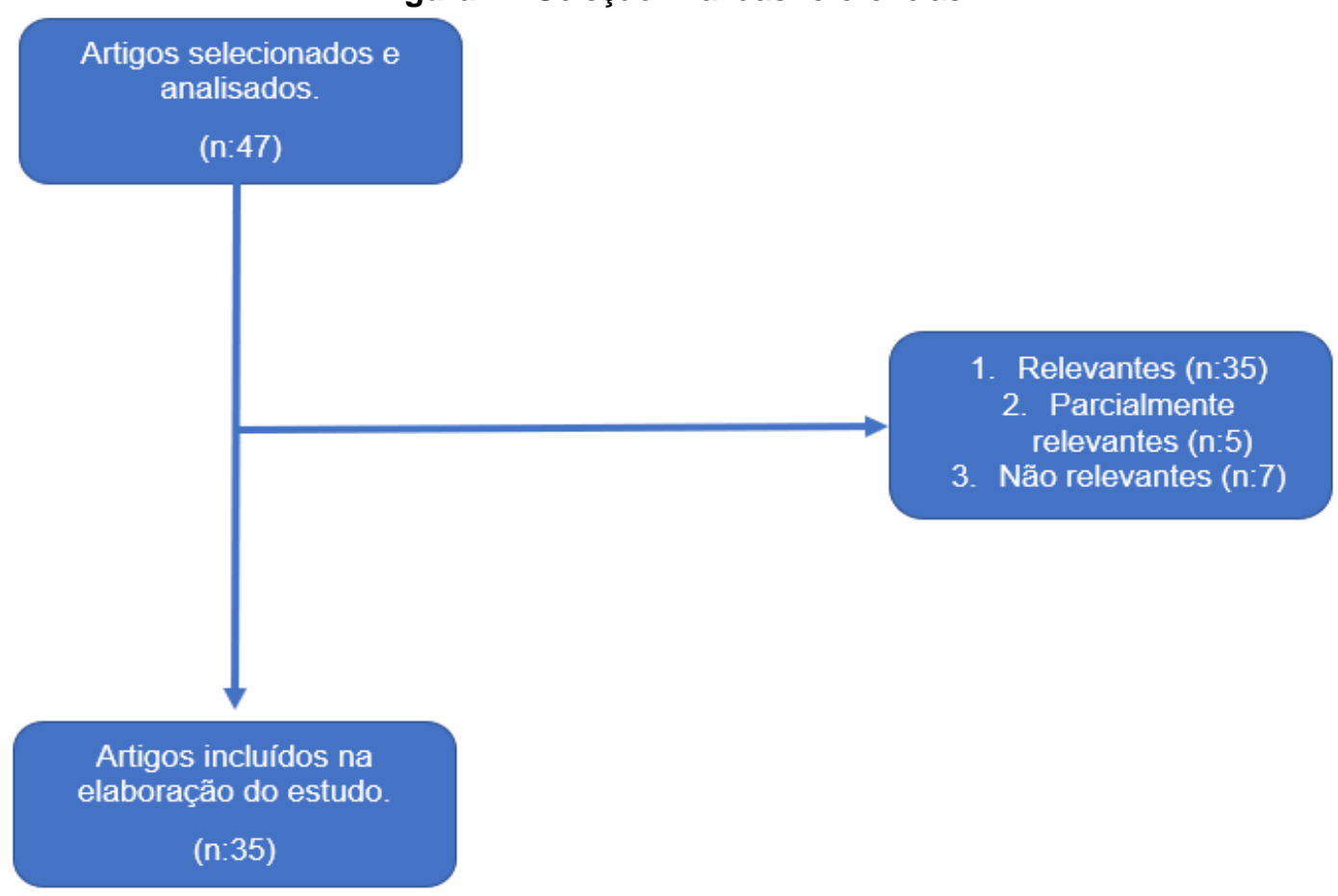

Fonte: Silva GA, et al., 2020. 


\section{DISCUSSÃO}

A DM2 é um importante fator de risco para o desenvolvimento de doenças de origem cardiovascular (ZINMAN B, et al., 2015), aumentando cerca de 2 a 4 vezes a probabilidade de eventos cardiovasculares em diabéticos, quando comparado à não diabéticos (ÅKERBLOM A, et al., 2019; VETTOR R, et al., 2017).

No diabético, o estado hiperglicêmico, a resistência insulínica dos tecidos, principalmente o cardíaco, e o depósito de gordura visceral são grandes fatores de risco para o desenvolvimento e agravamento de IC e eventos isquêmicos (VERMA S e MCMURRAY JJV, 2019). Apesar disso, a associação entre o controle glicêmico intensivo e diminuição das taxas de complicações e mortalidade cardiovascular é controversa, visto que determinados hipoglicemiantes estão associados com aumento na probabilidade de eventos cardiovasculares graves (ZINMAN B, et al., 2015).

Terapêuticas hipoglicemiantes que utilizem insulina exógena ou medicações que estimulem a secreção de insulina endógena, estão associadas a maiores taxas de mortalidade por IC em diabéticos com doenças cardiovasculares preexistentes. Tais medicações, através da elevação dos níveis de insulina, são capazes de aumentar a captação de substratos energéticos, como ácidos graxos e glicose pelo coração, consequentemente promovendo elevação na gênese de excretas lipídicas tóxicas, como diacilglicerol e ceramidas, e aumento do mecanismo de glicação, processos estes, altamente lesivos ao tecido cardíaco (K.RIGGS, et al., 2015; VETTOR R, et al., 2017).

Assim, o tratamento de pacientes com medicações que elevem os níveis séricos de insulina dos pacientes diabéticos promovem o aumento de mecanismos de injúria ao tecido cardíaco, sendo capazes de promover alterações estruturais e funcionais no coração. Esta linha de pensamento estimula o raciocínio de que 0 tratamento da diabetes deve se sustentar na excreção do substrato energético excedente e não no aumento de seu consumo (K.RIGGS, et al., 2015).

Além do controle glicêmico, estudos demonstraram que para maior efetividade na redução do risco cardiovascular na DM2, há necessidade da abordagem terapêutica de outras eventuais comorbidades existentes como HAS, dislipidemia, Índice de Massa Corpórea (IMC) elevado e tabagismo (PANCHOLIA AK, 2018).

Para o desenvolvimento de terapêuticas hipoglicemiantes mais modernas é necessário conhecimento do metabolismo e mecanismo regulatórios da glicose. Os rins e seu funcionamento adequado são fundamentais para a homeostase do metabolismo da glicose (PANCHOLIA AK, 2018). Além de exercerem papel importante na gliconeogênese, evitam a excreção de glicose na urina.

Diariamente, 160 a 180 gramas de glicose passam pelo processo de filtração renal e, conquanto não excedam esses valores, têm $100 \%$ do filtrado reabsorvido à circulação (FERRANNINI, et al., 2017). Em indivíduos que ultrapassam os valores limítrofes de reabsorção, como em diabéticos, há perda de glicose na urina, a glicosúria (ROCHA NA, et al., 2018).

Para possibilitar o mecanismo de reabsorção da glicose, o néfron conta com estruturas específicas transportadoras de glicose, os SGLT1 e os SGLT2. Tais transportadores, foram descobertos na década de 1970, localizados na membrana apical do túbulo renal (FOOTE C, et al., 2012) e com a função de possibilitar a reabsorção de glicose através de um processo mediado pela bomba de trifosfato adenosina (ATP) e do transporte ativo de sódio para o meio extracelular (PANCHOLIA AK, 2018).

O túbulo proximal é responsável pela reabsorção de $90 \%$ da glicose filtrada pelo glomérulo (PANCHOLIA AK, 2018; ROCHA NA, et al. 2018; BHARTIA M, et al. 2011). Esta porção do néfron é a que contém a maior concentração de SGLT2, que apesar de possuírem baixa afinidade possuem grande capacidade transportadora de glicose (FOOTE C, et al., 2012; K.RIGGS, et al., 2015; FERRANNINI, et al., 2017).

O restante da glicose não reabsorvida na porção proximal será reabsorvido pela porção distal do néfron (PANCHOLIA AK, 2018; ROCHA NA, et al., 2018; BHARTIA M, et al., 2011), mediado pelos SGLT1, receptores de alta afinidade e baixa capacidade de transporte da glicose (FOOTE C, et al., 2012; K.RIGGS, et al., 2015). Os SGLT1 estão presentes ainda nas microvilosidades intestinais possibilitando a reabsorção intestinal de glicose (FERRANNINI, et al., 2017). 
Após a reabsorção, a glicose encontra-se no espaço intracelular, em contato com a membrana basolateral da célula tubular, e mediada pelo transportador de glicose 2 (GLUT2), sofre um processo de difusão passiva, sendo reabsorvido para o sangue (PANCHOLIA AK., 2018).

Desta forma, desenvolveu-se os hipoglicemiantes inibidores da SGLT2, com mecanismo de ação independente da insulina e atua bloqueando de maneira parcial à moderada os receptores SGLT2 no néfron, impedindo 30-50\% da reabsorção de glicose por essas estruturas. Estima-se com isso a excreção de 60-100 gramas/dia de glicose, equivalente à $240-400 \mathrm{kcal} / \mathrm{dia}$, fato que além do controle glicêmico favorece à perda de peso corpóreo.

Observou-se ainda outros efeitos benéficos como redução da pressão arterial, ácido úrico e resistência insulínica. Seu uso também mostrou-se seguro, com baixo risco de hipoglicemia e outros efeitos colaterais indesejáveis (PANCHOLIA AK, 2018; HEERSPINK HJL, et al., 2016; RIGGS K, et al., 2015; FOOTE C, et al., 2012; PFEIFER M, et al., 2017; TAKENAKA T, et al., 2017; LEE G, et al., 2017).

As principais drogas representantes desta classe com estudos conduzidos para avaliação da influência sobre o risco cardiovascular, atendendo as normas da FDA (CEFALU WT, et al., 2018), foram a empaglifozina, dapaglifozina e canaglifozina.

$O$ estudo que forneceu as maiores informações e surpresas com a classe foi conduzido com a empaglifozina. Nomeado The Empagliflozin Cardiovascular Outcome Event Trial in Type 2 Diabetes Mellitus Patients-Removing Excess Glucose (EMPA-REG OUTCOMES) randomizado, duplo cego e avaliando medicamento versus placebo.

O desenvolvimento do estudo ocorreu em 590 locais, dispostos em 42 países, com a seleção de 7028 pacientes. Os critérios de inclusão foram, adultos maiores de 18 anos, portadores de DM2 e doença cardiovascular, IMC $<45$, hemoglobina glicada (HbA1c) entre $7-10 \%$, TFG maior ou igual a $30 \mathrm{ml} / \mathrm{min}$ e que não foram tratadas nas últimas 12 semanas com inibidores da SGLT2 (ZINMAN B, et al., 2015; FITCHETT D, et al., 2019).

Dos 7028 pacientes selecionados, 7020 foram divididos em três grupos distintos. Ao todo, 4687 pacientes foram tratados com a medicação, sendo 2345 com empaglifozina $10 \mathrm{mg}$ e $2342 \mathrm{com}$ empaglifozina 25mg, enquanto 2333 receberam placebo.

O estudo desenrolou-se por uma média de 2.6 anos, ocorrendo até que 691 participantes tivessem sido acometidos por morte de causa cardiovascular, IAM ou AVC não fatais, chamados de desfechos primários. $O$ estudo contou ainda com a avaliação de desfechos secundários, que incluíam os primários e adicionava hospitalização por angina instável (ZINMAN B, et al., 2015; FITCHETT D, et al., 2019; SATTAR N, et al., 2016).

Ao final, constatou-se que o uso da empaglifozina associou-se à redução da incidência de alterações cardiovasculares primárias e secundárias. O grupo da empaglifozina contou com incidência de alterações primárias de 10,5\%, contra 12,5\% do placebo. Quanto aos desfechos secundários, 12,8\% tiveram alterações com a medicação, enquanto 14,3\% com o placebo (ZINMAN B, et al., 2015; FITCHETT D, et al., 2019).

Outro dado surpreendente foi que com o emprego da empaglifozina, constatou-se redução de $38 \%$ da probabilidade de morte cardiovascular, 32\% por morte de quaisquer causas e 35\% de hospitalização por insuficiência cardíaca (SATTAR N, et al., 2016; VERMA S e MCMURRAY JJV, 2019).

O EMPA-REG OUTCOMES demonstrou ainda outros benefícios com o uso da empaglifozina. Constatouse diminuições da HbA1c entre 0,3\% a 0,8\% (HEERSPINK HJL, et al., 2016; SATTAR N, et al., 2016; SUISSA S, 2018; ZINMAN B, et al., 2015; SATTAR N, et al., 2016; PACHOLIA AK, 2018).

Valores pressórico sofreram alterações discretas (SATTAR N, et al., 2016), com diminuição de 3-5mmHg na Pressão Arterial Sistólica (PAS) e 2-3mmHg na Pressão Arterial Diastólica (PAD) sem alterações na frequência cardíaca (HEERSPINK HJL, et al., 2016; PACHOLIA AK, 2018), mecanismo possivelmente explicado devido a natriurese osmótica induzida pela glicosúria (FOOTE C, et al., 2012; VETTOR R, et al., 2017). 
A empaglifozina mostrou-se capaz de reduzir o IMC de maneira isolada (PACHOLIA AK, 2018; FOOTE C, et al., 2012). Perdas ponderais de 2-3kg, foram descritas com o uso da medicação, sobretudo nos 6 primeiros meses de tratamento, quiçá pela perda calórica induzida pela glicosúria (FOOTE C, et al., 2012; PACHOLIA AK, 2018).

Outro benefício observado está na diminuição dos níveis séricos de ácido úrico (ZINMAN B, et al., 2015; PACHOLIA AK, 2018). Valores hiperuricêmicos estão associados com maiores chances de HAS, doenças cardiovasculares e problemas renais. Os inibidores da SGLT2, através da glicosúria, estimulam a secreção de ácido úrico, causando depleção de 10-15\% dos níveis plasmáticos (HEERSPINK HJL, et al., 2016).

Quanto aos efeitos colaterais, foi elencado a hipótese de aumento na chance de Infecção do Trato Urinário (ITU), devido ao aumento do substrato para proliferação bacteriana possivelmente induzido pela glicosúria. No entanto, o EMPA-REG OUTCOME não demonstrou aumento do risco de ITU, ITU complicada e pielonefrite.

Não houve associação entre alterações eletrocardiográficas com a empaglifozina (RING A, et al., 2013), incidência aumentada de eventos adversos graves, episódios hipoglicêmicos, cetoacidose diabética, eventos tromboembólicos, fraturas ósseas, amputações, complicações por depleção de volume e insuficiência renal (ZINMAN B, et al., 2015).

O principal efeito adverso registrado foi o aumento de infecções genitais em homens e mulheres, sobretudo devido à Cândida sp. (FOOTE C, et al., 2012). Reportou-se incidência de 6,4\% com a medicação e de 1,8\% com o placebo (SATTAR N, et al., 2016) demonstrando risco aumentado de 9-18\% em mulheres, e metade desses valores para homens (FOOTE C, et al., 2012). Outros efeitos colaterais descritos foram o aumento do hematócrito e dos valores do HDL e LDL, ambos sem complicações relatadas (FOOTE C, et al., 2012).

Quanto à Canaglifozina, o estudo desenvolvido foi o Canaglifozin Cardiovascular Assessment Study (CANVAS), duplo-cego, randomizado e medicação versus placebo. Selecionou-se 4330 indivíduos, igualmente divididos e tratados com canaglifozina $100 \mathrm{mg}$, 300mg ou placebo (NEAL B, et al., 2017; KLUGER AY, et al., 2018).

Com a utilização da medicação houve redução em $14 \%$ no risco de eventos cardiovasculares, $13 \%$ de morte cardiovascular e 33\% de hospitalização por IC (YEHYA A e SADHU AR, 2018; PANCHOLIA AK, 2018). A PA também sofreu influências, com descrições de redução nos valores de 4,3-5,0mmHg na PAS e 2,4$2,5 \mathrm{mmHg}$ na PAD (PFEIFER M, et al., 2017). Negativamente, descreveu-se aumento no risco de amputações e câncer vesical, mas com necessidade de estudos determinando a real correlação entre os fatores (LYTVYN Y, et al., 2017; PFEIFER M, et al., 2017).

O estudo Dapagliflozin Effect on Cardiovascular Events-Trombolysis in Myocardial infarction 58 (DECLARE-TIMI 58), comparou os efeitos da Dapaglifozina versus placebo em pacientes com doença aterosclerótica estabelecida e IC.

Determinou-se risco $27 \%$ menor de hospitalização por IC e diminuição do risco cardiovascular em pacientes com e sem histórico de IC (LAM C, et al., 2019, KLUGER AY, et al., 2018), registrando ainda redução na PA, IMC e albuminúria (PETRYKIV S, et al., 2017).

Outros estudos também forneceram dados semelhantes aos principais estudos com inibidores da SGLT2 (MAZIDI M, et al., 2017). Em estudo conduzido na Noruega, Dinamarca e Suécia, comparou-se pacientes em uso de inibidores da SGLT2 com usuários de inibidores da DPP4, determinando que os glicosúricos tiveram maior redução no risco de IC, mortalidade geral e cardiovascular (PASTERNAK B, et al., 2019).

Em outro estudo, o CVD-REAL (Comparative Effectiveness of Cardiovascular Outcomes in New Users of Sodium-Glucose Cotransporter-2 Inhibitors), 309.056 pacientes foram selecionados em 6 países diferentes, comparando inibidores SGLT2 com outras classes hipoglicemiantes em relação as taxas de hospitalização por IC, mortalidade cardiovascular e geral.

Ao fim, evidenciou-se benefício para populações independentemente do risco cardiovascular, reduzindo $39 \%$ a incidência de internações por IC, $51 \%$ em mortalidade geral e $46 \%$ em mortes cardiovasculares com uso dos inibidores da SGLT2 (KOSIBOROD M, et al., 2017). 


\section{CONSIDERAÇÕES FINAIS}

A DM2 é conhecidamente um grande fator de risco para o desenvolvimento e agravamento de doenças cardiovasculares. Desta forma, a terapêutica desta comorbidade deve almejar, além do controle glicêmico, a diminuição do risco cardiovascular e de eventos cardiovasculares preocupantes. Com o fornecimento de dados através de inúmeros estudos com bom índice de confiabilidade, como o EMPA-REG OUTCOME, CANVAS e DECLARE-TIMI 58, constatou-se a capacidade dos inibidores da SGLT2 em reduzir o risco cardiovascular, promovendo diversas alterações, como diminuição dos níveis pressóricos, peso corpóreo, resistência insulínica, uricemia e deposição de gordura visceral, além de reduzirem substancialmente as taxas de hospitalização por IC, eventos isquêmicos, mortalidade geral e de origem cardiovascular. Desta maneira, os inibidores da SGLT2 mostram-se, atualmente, como uma das melhores opções terapêuticas disponíveis no mercado para o tratamento da DM2 e na redução do risco cardiovascular, devendo ter utilização cogitada e oferecida a todos os pacientes diabéticos tipo 2 .

\section{REFERÊNCIAS}

1. ÅKERBLOM A, et al. Effects of DAPAgliflozin on CARDiac substrate uptake, myocardial efficiency, and myocardial contractile work in type 2 diabetes patients - a description of the DAPACARD study. Upsal Journal of Medical Sciences. 2019; 124(1): 59-64.

2. BHARTIA M, et al. SGLT-2 Inhibitors in Development for Type 2 Diabetes Treatment. The Review of Diabetic Studies. 2011; 8(3): 348-354.

3. CEFALU WT. et al. Cardiovascular outcomes trials in type 2 diabetes: Where Do We Go From Here? Reflections From a Diabetes Care Editors' Expert Forum. Diabetes Care. 2018; 41(1): 14-21.

4. FERRANNINI E. Sodium-Glucose Co-transporters and Their Inhibition: Clinical Physiology. Cell Metabolism. 2017; 26(1): 27-38.

5. FISMAN EZ, TENENBAUM A. Antidiabetic treatment with gliptins: Focus on cardiovascular effects and outcomes. Cardiovascular Diabetology. 2015; 14(1)1-13.

6. FITCHETT D, et al. Empagliflozin Reduced Mortality and Hospitalization for Heart Failure Across the Spectrum of Cardiovascular Risk in the EMPA-REG OUTCOME Trial. Circulation. 2019; 139(11): 1384-1395.

7. FOOD AND DRUG ADMINISTRATION. Victoza (Liraglutide) Approval Letter. FDA. 2010; p 1-10.

8. FOOTE C, et al. Effects of SGLT2 inhibitors on cardiovascular outcomes. Diabetes and Vascular Disease Research. 2012; 9(2): 117-123.

9. GILBERT RE, CONNELLY KA. Reduction in the incidence of myocardial infarction with sodium-glucose linked cotransporter-2 inhibitors: Evident and plausible. Cardiovascular Diabetology. 2019; 18(1): 4-6.

10. HEERSPINK HJL, et al. Sodium Glucose Cotransporter 2 Inhibitors in the Treatment of Diabetes Mellitus: Cardiovascular and Kidney Effects, Potential Mechanisms, and Clinical Applications. Circulation. 2016; 134(10): 752-772.

11. INTERNATIONAL DIABETES FEDERATION. IDF Diabetes Atlas, 2019; 72-73.

12. RIGGS K, et al. The Use of SGLT-2 Inhibitors in Type 2 Diabetes and Heart Failure. Metabolic Syndrome and Related Disorders. 2015; 13(7): 292-297.

13. KAUL S. Mitigating cardiovascular risk in type 2 diabetes with antidiabetes drugs: A review of principal cardiovascular outcome results of EMPA-REG OUTCOME, LEADER, and SUSTAIN-6 trials. Diabetes Care. 2017; 40(7): 821-831.

14. KLUGER AY, et al. Cardiorenal Outcomes in the CANVAS, DECLARE-TIMI 58, and EMPA-REG OUTCOME Trials: A Systematic Review. Reviews in cardiovascular medicine. 2019; 19(2): 41-49

15. KOSIBOROD M, et al. Lower risk of heart failure and death in patients initiated on sodium-glucose cotransporter-2 inhibitors versus other glucose-lowering drugs: The CVD-REAL study (Comparative Effectiveness of Cardiovascular Outcomes in New Users of Sodium-Glucose Cotransporter-2 Inhibitors). Circulation. 2017; 136(3): 249-259.

16. LAM C, et al. SGLT-2 Inhibitors in Heart Failure: Current Management, Unmet Needs, and Therapeutic Prospects. Journal of the American Heart Association. 2019; 8(20): 1-12.

17. LEE G, et al. Comparative effectiveness of oral antidiabetic drugs in preventing cardiovascular mortality and morbidity: A network meta-analysis. PLoS ONE. 2017; 12(5): 1-16.

18. LEY SH, et al. Risk Factor for Type 2 Diabetes Mellitus. Diabetes in America, 3rd Edition, 2015; 13,1-13,5.

19. LYTVYN Y, et al. Sodium glucose cotransporter-2 inhibition in heart failure: Potential mechanisms, clinical applications, and summary of clinical trials. Circulation. 2017; 136(17): 1643-1658.

20. MANN JFE, et al. Effects of liraglutide versus placebo on cardiovascular events in patients with type 2 diabetes mellitus and chronic kidney disease: Results from the LEADER trial. Circulation. 2018; 138(25): 2908-2918.

21. MAZIDI M, et al. Effect of sodium-glucose cotransport-2 inhibitors on blood pressure in people with type 2 diabetes mellitus: A systematic review and meta-analysis of 43 randomized control trials with 22528 patients. Journal of the American Heart Association. 2017; 6(6): 1-12.

22. NEAL B, et al. Optimizing the analysis strategy for the CANVAS Program: A prespecified plan for the integrated analyses of the CANVAS and CANVAS-R trials. Diabetes, Obesity and Metabolism. 2017; 19(7): 926-935. 
23. OLIVEIRA JEP, et al. Diretrizes da Sociedade Brasileira de Diabetes, 2017; $12-13$.

24. PANCHOLIA AK. Sodium-glucose cotransporter-2 inhibition for the reduction of cardiovascular events in high-risk patients with diabetes mellitus. Indian Heart Journal. 2018; 70(6): 915-921.

25. PASTERNAK B, et al. Use of sodium glucose cotransporter 2 inhibitors and risk of major cardiovascular events and heart failure: Scandinavian register based cohort study. The Bmj. 2019; 366(1): 1-11.

26. PFEIFER M, et al. Effects of canagliflozin, a sodium glucose co-transporter 2 inhibitor, on blood pressure and markers of arterial stiffness in patients with type 2 diabetes mellitus: A post hoc analysis. Cardiovascular Diabetology. 2017; 16(1): 1-9.

27. PTRYKIV S, et al. Differential effects of dapagliflozin on cardiovascular risk factors at varying degrees of renal function. Clinical Journal of the American Society of Nephrology. 2017; 12(5): 751-759.

28. RIGGS K, et al. The use of SGLT-2 inhibitors in type 2 diabetes and heart failure. Metabolic Syndrome and Related Disorders. 2015; 13(7): 292-297.

29. RING A, et al. The sodium glucose cotransporter 2 inhibitor empagliflozin does not prolong QT interval in a thorough QT (TQT) study. Cardiovascular Diabetology. 2013; 12(1): 1-11.

30. ROCHA NA, et al. Effects of sodium glucose co-transporter 2 inhibitors on the kidney. Diabetes and Vascular Disease Research. 2018; 15(5): 375-386.

31. SATTAR N, et al. SGLT2 Inhibition and cardiovascular events: why did EMPA-REG Outcomes surprise and what were the likely mechanisms?. Diabetologia. 2016; 59(7): 1333-1339.

32. SCHNELL O, et al. Updates on cardiovascular outcome trials in diabetes. Cardiovascular Diabetology. 2017;16(1): 1-13.

33. SOCIEDADE BRASILEIRA DE DIABETES. Medicamentos orais no tratamento do diabetes mellitus: como selecioná-los de acordo com as características clínicas dos pacientes. Diretrizes SBD, 2014; 48-52.

34. STOORGAARD H, et al. Benefits and Harms of Sodium Glucose Cotransporters 2 Inhibitors in Patients with Type 2 Diabetes: A Systematic Review and Meta-Analysis. PLoS ONE. 2016; 11(11): 1-23.

35. SUISSA S. Mortality Reduction in EMPA-REG OUTCOME Trial: Beyond the Antidiabetes Effect. Diabetes Care. 2018; $41(2):$ 219-223.

36. TAKENAKA T, et al. Sodium-glucose co-transporter type 2 inhibitors reduce evening home blood pressure in type 2 diabetes with nephropathy. Diabetes and Vascular Disease Research. 2017; 14(3): 258-261.

37. VERMA S, MCMURRAY JJV. The Serendipitous Story of SGLT2 Inhibitors in Heart Failure. Circulation. 2019; 139(22): 2537-2541.

38. VETTOR R, et al. The cardiovascular beneficits of Empagliflozin SGLT2-dependent and -independent effects. Diabetologia. 2017; 60(3): 395-398.

39. YEHYA A, SADHU AR. New Therapeutic Strategies for Type 2 Diabetes. Methodist Debakey Cardiovasc Journal. 2018; 14(4): 281-288.

40. ZINMAN B, et al. Empagliflozin, Cardiovascular Outcomes, and Mortality in Type 2 Diabetes. New England Journal of Medicine. 2015; 157(34): 2117-2128. 